\title{
Vortex Dynamics Equation in Type-II Superconductors in a Temperature Gradient
}

\author{
R. Vega Monroy* and J. Sarmiento Castillo \\ Facultad de Ciencias Básicas. Universidad del Atlántico Km. 7, Via a Pto. Colombia, Barranquilla, Colombia \\ D. Puerta Torres \\ Facultad de Ciencias Exactas. Universidad de Cartagena Plaza de la Artillería N. 30-84, Cartagena, Colombia
}

(Received on 27 August, 2010)

\begin{abstract}
In this work we determined a vortex dynamics equation in a temperature gradient in the frame of the time dependent Ginzburg-Landau equation. In this sense, we derived a local solvability condition, which governs the vortex dynamics. Also, we calculated the explicit form for the force coefficients, which are the keys for the understanding of the balance equation due to vortex interactions with the environment.
\end{abstract}

Keywords: Type-II Superconductors; Vortex Equation; Vortex Balance Equation.

\section{INTRODUCTION}

In type-II superconductors, the vortices are the ones in charge for the magnetic properties of these systems since every vortex carries a magnetic flux quantum. In the last years the interest in the vortex motion is associated to many non peculiar properties in HTSC not found in conventional typeII superconductors. In particular, one of the most important effects encountered in HTSC is the Hall anomaly [1,2]. In this sense, it is known that, under the action of the Lorentzs force vortices acquire a movement and therefore losses appear in the superconducting state. The equation of motion, which governs the vortex dynamics in type-II superconductors, has been subject to a great amount of works, which have helped us to understand this phenomenon in these systems. In addition, the interest in the vortex motion is emphasized by the responsibility of this dynamics in a great variety of transport phenomena in type II superconductors. Generally, the vortex dynamics has been considered on the hydrodynamical two fluid model $[3,4]$, where the relative motion between the superfluid and the vortex generates the Magnus force. Next, the normal component reacts to this motion, producing the longitudinal viscous drag force and the transversal Iordanski's force, which are the two components of the medium force. An attempt to describe the vortex dynamics was done by Dorsey [5] in the frame of the time dependent Ginzburg-Landau equation following previous developments of Gorkov and Kopnin [6]. In this work, Dorsey formulates a solubility condition through with a vortex equation can be obtained. Today, there are several attempts to construct a unified theory about the vortex motion. Some approximations use the sophisticated many body formalism [7,8,9]. Other authors apply a simpler theory based on the kinetic Boltzmann equation to study the dynamic behavior of the vortex structures [10], but so far the vortex dynamics is an open question for the solid state physics community. The purpose of the present work is to contribute to a better understanding of the fascinating phenomenon of the vortex motion. In this connection, the goal of this paper is to determine a vortex dynamics equation in a temperature gradient in the frame of the time dependent Ginzburg-Landau equation. Such kind

*Electronic address: rvega@uniatlantico.edu.co of equation has been introduced in a heuristic way in many works [11] to satisfy experimental data $[12,13,14]$.

The paper is organized as follows: In Section 2, we have obtained the basics equations of the work. In Section 3, some dynamic coefficients are calculated and finally in Section 4 we summarized the main results.

\section{BASIC EQUATIONS}

The present analysis follows the works done by Dorsey [5] in order to obtain the equation, which describes the vortex dynamics. Let us write the dimensionless time dependent Ginzburg-Landau equation for the complex order parameter in the form:

$$
\gamma\left[\frac{\partial}{\partial t}+i \phi\right] \psi=\left[\frac{\vec{\nabla}}{\kappa}-i \vec{A}\right]^{2} \psi+\psi-|\psi|^{2} \psi .
$$

In the above equation $\gamma$ is the dimensionless relaxation time, К is the Ginzburg-Landau parameter, $\Phi$ and $\vec{A}$ are the electric potential and the magnetic vector respectively. The order parameter $\psi$ in terms of the amplitude $f(\vec{r}, t)$ and the fase $\chi(\vec{r}, t)$ can be represented as follows $\psi(\vec{r}, t)=f(\vec{r}, t) \exp [i \chi(\vec{r}, t)]$. The relaxation time has a complex character and can be written

$$
\gamma=\gamma_{1}+i \gamma_{2}
$$

The appearance of the imaginary part in the previous expression is a necessary condition for the gauge invariance conservation of equation (1) [10]. Relaxation processes that entail to dispersion in the vortex dynamics are of two types: the first is associated to the Bardeen- Stephens mechanism of dissipation and the second process is an intrinsic relaxation mechanism that governs the approach of the order parameter to its equilibrium state due to variations in the chemical potential. This mechanism is associated to the change of the order parameter in time because the electrons are forced to pair and de-pair with respect to different potentials due to the vortex motion. In consequence, this processe is the responsible that the relaxation time acquires a complex character as was shown by Kopnin [10] and it is framed in the parameter $\gamma_{2}$

$$
\gamma_{2}=-\frac{\hbar}{2 \lambda} \frac{\partial v}{\partial \mu}
$$


where $v$ and $\mu$ are the density of states and the chemical potential respectively. Introducing $\psi$ and $\gamma$ in the expression (1) and in addition, introducing the invariant forms for the magnetic vector and the scalar potential

$$
\vec{Q}=\vec{A}-\frac{1}{\kappa} \vec{\nabla} \chi, \quad P=\Phi+\frac{\partial \chi}{\partial t},
$$

one can obtain two equations, the first for the real part and the other one for the imaginary part as follows:

$$
\begin{gathered}
\gamma_{1} \frac{\partial f}{\partial t}-\gamma_{2} f P=\frac{1}{\kappa^{2}} \vec{\nabla}^{2} f+Q^{2} f-f-f^{3}, \\
\gamma_{1} f P-\gamma_{2} \frac{\partial f}{\partial t}+\frac{2}{\kappa} \vec{Q} \cdot \vec{\nabla} f+\frac{f}{\kappa} \vec{\nabla} \cdot \vec{Q}=0 .
\end{gathered}
$$

To form a closed system of equations, we need to derive an equation for the magnetic vector. In this connection, the dimensionless equations for the superconducting and normal current are:

$$
\begin{gathered}
\vec{J}_{s}=\frac{1}{2 \kappa i}(\psi * \vec{\nabla} \psi-\psi \vec{\nabla} \psi *)-|\psi|^{2} \vec{A}=-f^{2} \vec{Q}, \\
\vec{J}_{n}=\sigma^{(n)}\left(-\frac{1}{\kappa} \vec{\nabla} P-\frac{\partial \vec{Q}}{\partial t}\right)+\frac{1}{\kappa} b^{(n)} \vec{\nabla} T,
\end{gathered}
$$

where $\sigma^{(n)}$ and $b^{(n)}$ are the electric and thermoelectric conductivities in the normal state respectively. In this sense, the dimensionless equation for the magnetic vector is:

$$
\vec{\nabla} \times \vec{\nabla} \times \vec{Q}=\sigma^{(n)}\left(-\frac{1}{\kappa} \vec{\nabla} P-\frac{\partial \vec{Q}}{\partial t}\right)+\frac{1}{\kappa} b^{(n)} \vec{\nabla} T-f^{2} \vec{Q} .
$$

Now, to find an equation for the potential $P$, one can use the vector relation $\vec{\nabla} \cdot\left(f^{2} \vec{Q}\right)=2 f \vec{\nabla} f \cdot \vec{Q}+f^{2} \vec{\nabla} \cdot \vec{Q}$, so that counting the fact that $\vec{\nabla} \cdot\left(\vec{J}_{s}+\vec{J}_{n}\right)=0$, i.e.

$$
\begin{aligned}
\vec{\nabla} \cdot\left(\vec{J}_{s}+\vec{J}_{n}\right)= & -\vec{\nabla} \cdot\left(f^{2} \vec{Q}\right)+\vec{\nabla} \cdot\left[\sigma^{(n)}\left(-\frac{1}{\kappa} \vec{\nabla} P-\frac{\partial \vec{Q}}{\partial t}\right)\right]+ \\
& +\frac{1}{\kappa} \vec{\nabla} \cdot\left(b^{(n)} \vec{\nabla} T\right)=0
\end{aligned}
$$

we obtain from equations (5) and (6) the following relation:

$$
\begin{aligned}
& \frac{1}{\kappa} \vec{\nabla} \cdot\left[\sigma^{(n)}\left(-\frac{1}{\kappa} \vec{\nabla} P-\frac{\partial \vec{Q}}{\partial t}\right)\right] \\
& +\frac{1}{\kappa} \vec{\nabla} \cdot\left(b^{(n)} \vec{\nabla} T\right)+\gamma_{1} f^{2} P+\gamma_{2} f \frac{\partial f}{\partial t}=0 .
\end{aligned}
$$

The above equation together with relations (3) and (7) will allow to arrive at the solvability condition for the equation that determines the vortex dynamics in type II superconductors.

On the other hand, locally in the vortex, the order parameter differs from the value in the bulk of superconductor so that a deviation appears in the order parameter and the potentials. In this connection, the quantities $f$ and $\vec{Q}$ are expanded

$$
f=f_{0}+f_{1}, \quad \vec{Q}=\vec{Q}_{0}+\vec{Q}_{1},
$$

where $f_{0}$ and $f_{1}$ are the amplitudes of the order parameter associated to the equilibrium and non equilibrium states respectively. In addition, in the vortex motion it is possible to consider that vortices move independently in the first approximation of the limit $B \ll H c 2$, so that one can find an equation of motion for every individual vortex in presence of a temperature gradient. In this limit, assuming that the vortices move uniformly, the quantities $f, \vec{Q}$ and P are functions solely of $\vec{r}-\vec{v}_{L} t$, where $\vec{r}$ is the electron position vector and $\vec{v}_{L}$ is the vortex velocity. Thus, the temporary derivative can be written in terms of spatial derivative by means of $\frac{\partial}{\partial t}=-\vec{v}_{L} \cdot \vec{\nabla}$, so if we introduce this relation in (3), (7) and (9), we obtain the following set of equations for the equilibrium state:

$$
\begin{gathered}
\frac{1}{\kappa^{2}} \nabla^{2} f_{0}+Q_{0}^{2} f_{0}-f_{0}-f_{0}^{3}=0, \\
\vec{\nabla} \times \vec{\nabla} \times \vec{Q}_{0}+f_{0}^{2} \vec{Q}_{0}=0,
\end{gathered}
$$

being

$$
\overrightarrow{J_{0}}=-f_{0}^{2} \vec{Q}_{0}
$$

the equilibrium current density. For the non equilibrium state we have the equations

$\frac{1}{\kappa^{2}} \nabla^{2} f_{1}+Q_{0}^{2} f_{1}-2 f_{0} \vec{Q}_{0} \vec{Q}_{1}-f_{1}-3 f_{0}^{2} f_{1}+\gamma_{2} P f_{0}=-\gamma_{1} f_{v}$,

$$
\begin{aligned}
& \vec{\nabla} \times \vec{\nabla} \times \vec{Q}_{0}=-f_{0}^{2} \vec{Q}_{1}-2 f_{0} f_{1} \vec{Q}_{0}+\sigma^{(n)}\left(-\frac{1}{\kappa} \vec{\nabla} P+\right. \\
& \left.+\vec{Q}_{v}\right)+\frac{1}{\kappa} b^{(n)} \vec{\nabla} T=\vec{J}_{1 s}+\vec{J}_{1 n}=\vec{J}_{1}
\end{aligned}
$$

where we have considered the fact that $f_{0} \gg f_{1}, \vec{Q}_{0} \gg \vec{Q}_{1}$ and the linear approximation of the deviation. In addition we introduced the quantities

$$
f_{v} \equiv \vec{v}_{L} \cdot \vec{\nabla} f_{0}, \quad \vec{Q}_{v} \equiv\left(\vec{v}_{L} \cdot \vec{\nabla}\right) \vec{Q}_{0}
$$

The Ginzburg-Landau equations are translational invariant, and therefore $f_{0}(\vec{r}+\vec{d})$ and $\vec{Q}_{0}(\vec{r}+\vec{d})$ are the solutions to the equation (10), being $\vec{d}$ an arbitrary translation vector. If we expand in Taylors series these two quantities, the linear equations (13) and (14), without the nonhomogenous terms, can be written in the form

$$
\begin{aligned}
& \frac{1}{\kappa^{2}} \nabla^{2} f_{d}+f_{d} Q_{0}^{2}-2 f_{0} \vec{Q}_{0} \cdot \vec{Q}_{d}-f_{d}-3 f_{0}^{2} f_{d}=0, \\
& \vec{\nabla} \times \vec{\nabla} \times \vec{Q}_{0}=f_{0}^{2} \vec{Q}_{d}-2 f_{0} f_{d} \vec{Q}_{0}=\vec{J}_{d} \equiv(\vec{d} \cdot \vec{\nabla}) \vec{J}_{0},
\end{aligned}
$$

where we introduced the parameters $f_{d} \equiv \vec{d} \cdot \vec{\nabla} f_{0}$ and $\vec{Q}_{d} \equiv$ $(\vec{d} \cdot \vec{\nabla}) \vec{Q}_{0}$. In order to derive the solvability condition one can integrate the equations (13) and (16) over a cylindrical area. Using the in the plane Gauss theorem and neglecting superficial contributions, the following equations are obtained:

$$
f_{1} Q_{0}^{2}-2 f_{0} \vec{Q}_{0} \cdot \vec{Q}_{1}-f_{1}-3 f_{0}^{2} f_{1}+\gamma_{2} P f_{0}+\gamma_{1} f_{v}=0,
$$




$$
f_{d} Q_{0}^{2}-2 f_{0} \vec{Q}_{0} \cdot \vec{Q}_{d}-f_{d}-3 f_{0}^{2} f_{d}=0 .
$$

Now we multiply the equation (18) by $f_{d}$ and the equation (19) by $f_{1}$. Next both equations are combined to obtain the following expression:

$$
\int d^{2} r\left(2 f_{0} f_{d} \vec{Q}_{0} \cdot \vec{Q}_{1}-2 f_{0} f_{1} \vec{Q}_{0} \cdot \vec{Q}_{d}+\gamma_{2} f_{0} f_{d} P+\gamma_{1} f_{v} f_{d}\right)=0 .
$$

Multiplying the quantity $\vec{J}_{1 s}$ by $\vec{Q}_{d}$, and the quantity $\vec{J}_{d}$ by $\vec{Q}_{1}$, then these two expressions are subtracted each other and replacing in the equation (20) to obtain:

$$
\int d^{2} r\left(\vec{J}_{d} \cdot \vec{Q}_{1}-\vec{J}_{1 s} \cdot \vec{Q}_{d}\right)=-\int d^{2} r\left(\gamma_{2} P f_{0} f_{d}+\gamma_{1} f_{v} f_{d}\right) .
$$

In order to simplify, in the $\kappa \gg 1$ limit, from equations (2) and the expression for $\vec{Q}_{d}$, we have $\vec{Q}_{d} \approx-\vec{\nabla} \chi_{d} / \kappa$ and $\vec{Q}_{1} \approx$ $-\vec{\nabla} \chi_{1} / \kappa$. Inserting the above expressions in the left side of the equation (21) we obtain:

$$
\frac{1}{\kappa} \int d^{2} r\left(\vec{J}_{d} \cdot \vec{\nabla} \chi_{1}-\vec{J}_{1 s} \kappa \vec{\nabla} \chi_{d}\right)=\int d^{2} r\left(\vec{J}_{1 s} \kappa \vec{Q}_{d}-\vec{J}_{d} \kappa \vec{Q}_{1}\right) .
$$

Now considering the vector relation $\vec{\nabla} \cdot(\alpha \vec{A})=\vec{A} \cdot \vec{\nabla} \alpha+\alpha \vec{\nabla}$. $\vec{A}$ and in addition, $\vec{\nabla} \cdot \vec{J}_{d}=0$, we can apply the in the plane divergence theorem as follows:

$$
\begin{gathered}
\int d^{2} r\left(\vec{J}_{1 s} \cdot \vec{Q}_{d}-\vec{J}_{d} \cdot \vec{Q}_{1}\right)=-\frac{1}{\kappa} \int d^{2} r\left(\vec{J}_{1 s} \cdot \vec{\nabla} \chi_{d}-\vec{J}_{d} \cdot \vec{\nabla} \chi_{1}\right) \\
=-\frac{1}{\kappa} \int d \vec{S} \cdot\left[\vec{J}_{1 s} \chi_{d}-\vec{J}_{d} \chi_{1}\right]+\frac{1}{\kappa} \int d^{2} r \chi_{d} \vec{\nabla} \cdot \vec{J}_{1 s} .
\end{gathered}
$$

On the other hand, inside the vortex, the total current density satisfies the continuity equation:

$\vec{\nabla} \cdot \vec{J}=\vec{\nabla} \cdot\left(\vec{J}_{1 n}+\vec{J}_{1 s}+\vec{J}_{0}\right)=-\frac{\partial \rho}{\partial t}=\vec{v}_{L} \cdot \vec{\nabla} \rho=\vec{v}_{L} \cdot\left(\vec{\nabla} \rho_{n}+\vec{\nabla} \rho_{s}\right)$,

where we have considered that the total charge density is $\rho=$ $\rho_{n}+\rho_{s}$, being $\rho_{n}$ and $\rho_{s}$ the normal and superconducting charge densities respectively. In this way, from equation (11) we have:

$$
\vec{\nabla} \cdot \overrightarrow{J_{0}}=0
$$

So that the expression (24) take the form:

$$
\begin{aligned}
\vec{\nabla} \cdot \vec{J}_{1 s}=-\vec{\nabla} \cdot \vec{J}_{1 n}+\vec{v}_{L} \cdot \vec{\nabla} \rho_{n}+ & \vec{v}_{L} \cdot \vec{\nabla} \rho_{s}=-\vec{\nabla}\left(\vec{v}_{L} \cdot \rho_{n}\right) \\
& +\vec{v}_{L} \cdot \vec{\nabla} \rho_{n}+\vec{v}_{L} \cdot \vec{\nabla} \rho_{s} .
\end{aligned}
$$

Replacing the expressions (21) and (25) in the equation (23) and assuming that $\vec{v}_{n}$ homogeneous, we have is

$$
\begin{aligned}
\frac{1}{\kappa} \int\left[\vec{J}_{1 s} \chi_{d}-\vec{J}_{d} \chi_{1}\right] \cdot d \vec{S} & \\
=-\int\left(\gamma_{2} P f_{0} f_{d}-\gamma_{1} f_{v} f_{d}\right) d^{2} r & +\frac{1}{\kappa} \int d^{2} r \chi_{d}\left(\vec{v}_{L}-\vec{v}_{n}\right) \cdot \vec{\nabla} \rho_{n} \\
& +\frac{1}{\kappa} \int d^{2} r \chi_{d} \vec{v}_{L} \cdot \vec{\nabla} \rho_{s} .
\end{aligned}
$$

The equation (26) is the local solvability condition for the vortex motion. Also it is of the linear order in the vortex velocity. If this condition is not fulfilled, then the non homogenous equations do not have solution. The expression (26) contains an original contribution of this paper and will allow determining the equation that governs the vortex dynamics in type II superconductors in presence of a temperature gradient. The solvability condition obtained in the present work differs from Dorsey's expression [5] by the thermal flow force due to the temperature gradient and also by normal contributions (which give a right relation of the involved dynamic parameters) in the vortex dynamics.

In order to derive the vortex dynamics equation, it is necessary to introduce a coordinate system. In this sense, as much the applied temperature gradient as the transport current are oriented in $\mathrm{x}$ direction. The magnetic field is directed along the $\mathrm{z}$-axis. The vortex velocity vector and the displacement vector $\vec{d}$ form angles $\theta_{H}$ and $\phi$ with the $\mathrm{x}$ axis respectively. The geometry of the problem is pictured in Figure 1.

From Figure 1 we have:

$$
\vec{J}_{t}=J_{t}\left[\cos \theta \hat{e}_{r}-\sin \theta \hat{e}_{\theta}\right]
$$

$$
\vec{\nabla} T=\nabla T\left[\cos \theta \hat{e}_{r}-\sin \theta \hat{e}_{\theta}\right]
$$

$$
\begin{gathered}
\vec{v}_{L}=v_{L}\left[\sin \left(\theta-\theta_{H}\right) \hat{e}_{r}+\cos \left(\theta-\theta_{H}\right) \hat{e}_{\theta}\right], \\
\vec{d}=d\left[\cos (\theta-\phi) \hat{e}_{r}+\sin (\theta-\phi) \hat{e}_{\theta}\right]
\end{gathered}
$$

The differential equation for the scalar potential $P$ acquires the following form:

$$
\begin{gathered}
\frac{\sigma_{x x}^{(n)}}{k^{2}} \nabla^{2} P-\gamma_{1} P f_{0}^{2}= \\
{\left[\gamma_{2} f_{0} \frac{d f_{0}}{d r}-\frac{\sigma_{x y}^{(n)}}{k} \frac{d h_{0}}{d r}\right] v_{L} \sin \left(\theta-\theta_{H}\right)+\frac{b_{x x}^{(n)}}{k^{2}} \nabla^{2} T \cos \theta .}
\end{gathered}
$$

In the above equation $\vec{h}_{0}=\vec{\nabla} \times \vec{Q}_{0}$. It is possible to determine the form of the solution to the equation (31) considering the limit $\mathrm{r} \rightarrow 0$, then $\chi \rightarrow \theta$. Therefore, from the equation (2) we have $P \approx-\frac{\partial \chi}{\partial t} \approx \vec{v}_{L} \cdot \vec{\nabla} \theta=\frac{1}{r} \hat{e}_{\theta}$.

In general way, the solution to the non homogenous differential equation (31) takes the form:

$$
P(\vec{r})=v_{L} p_{1}(\vec{r}) \cos \left(\theta-\theta_{H}\right)+v_{L} p_{2}(\vec{r}) \sin \left(\theta-\theta_{H}\right)+p_{3}(\vec{r}),
$$

where $p_{1}$ is the solution to the homogenous equation (31), $p_{2}$ and $p_{3}$ are particular solutions to the nonhomogenous equation. From the previous expression, we have that, $p_{1}, p_{2}$ and $p_{3}$ satisfy the differential equations:

$$
\begin{gathered}
\frac{\sigma_{x x}^{(n)}}{\kappa^{2}} \nabla^{2} p_{1}-\gamma_{1} f_{0}^{2} p_{1}=0 \\
\frac{\sigma_{x x}^{(n)}}{\kappa^{2}} \nabla^{2} p_{2}-\gamma_{1} f_{0}^{2} p_{2}=\gamma_{2} f_{0} \frac{d f_{0}}{d r}-\frac{\sigma_{x y}^{(n)}}{\kappa} \frac{d h_{0}}{d r},
\end{gathered}
$$




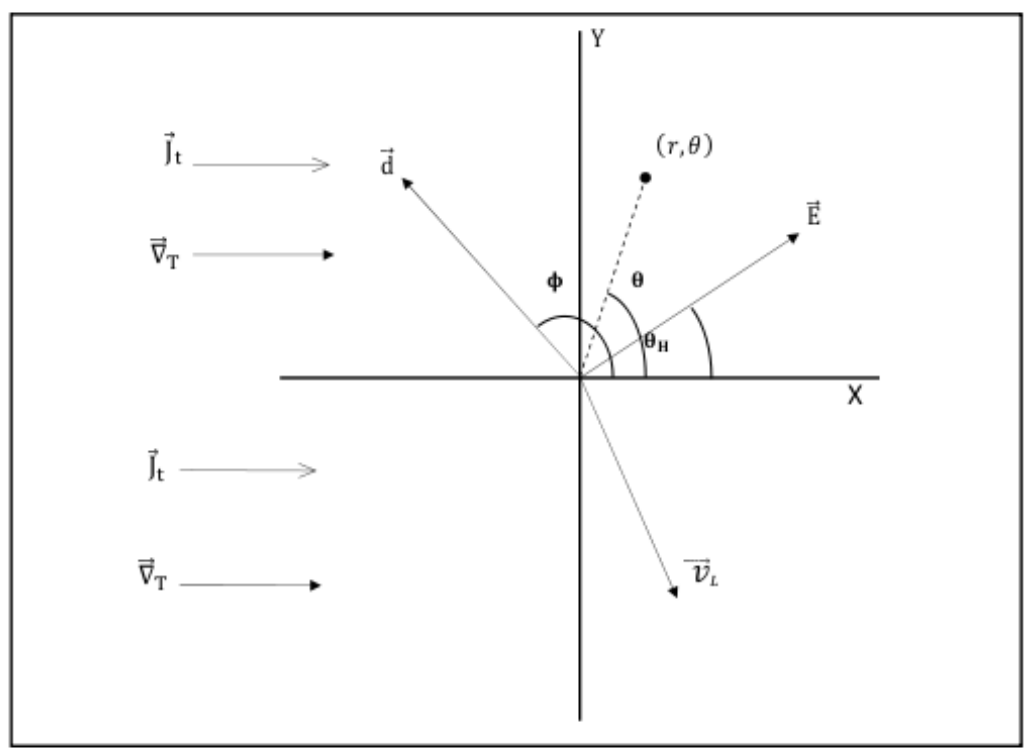

FIG. 1: Geometry of the system

$$
\frac{\sigma_{x x}^{(n)}}{\kappa^{2}} \nabla^{2} p_{3}-\gamma_{1} f_{0}^{2} p_{3}=\frac{b_{x x}^{(n)}}{\kappa^{2}} \nabla^{2} T \cos \theta .
$$

As $\vec{\nabla} T$ acts on normal electrons for small values of $r(r \rightarrow 0)$ then $f_{0}^{2} \rightarrow 0$ and for high values of $r$ superelectrons do not feel the presence of the temperature gradient, reason why in the equation (35) the second term of the left side can be neglected, having the solution (32) the form:

$$
\begin{aligned}
P(\vec{r})= & \vec{v}_{L} p_{1}(\vec{r}) \cos \left(\theta-\theta_{H}\right)+ \\
& +\vec{v}_{L} p_{2}(\vec{r}) \sin \left(\theta-\theta_{H}\right)-\varepsilon|\vec{\nabla} T| r \cos \theta,
\end{aligned}
$$

where the parameter $\varepsilon=\frac{b_{x x}^{(n)}}{\sigma_{x x}^{(n)}}$ was introduced.

\section{VORTEX DYNAMICS EQUATION}

The solvability condition can be evaluated solving the equation (26). The surface integral can be expressed in terms of the applied transport current at the borders, where

$$
\vec{J}_{1 s}(r=\infty, \theta)=\vec{J}_{t}, \vec{J}_{d} \cdot \hat{e}_{r}=d \sin \frac{(\theta-\phi)}{\kappa r^{2}},
$$

$$
\chi_{1}=\kappa J_{t} r \cos \theta, \chi_{d} \equiv \vec{d} \cdot \vec{\nabla} \theta=d \sin \frac{(\theta-\phi)}{r},
$$

so that the first integral to evaluate is the only one in the left side of the equation (26), where we use the formulae for the transport current $\vec{J}_{t}$ and $\chi_{d}$ in cylindrical coordinates shown in the equations (27) and the trigonometric equality $\sin (\theta-\phi)=\sin \theta \cos \phi-\cos \theta \sin \phi$,

$$
\begin{aligned}
\frac{1}{\kappa} \int d \vec{S} \cdot\left[\vec{J}_{1 s} \chi_{d}-\vec{J}_{d} \chi_{1}\right]=\frac{1}{\kappa} \int\left(r d \theta \hat{e}_{r}\right) \cdot\left(\overrightarrow{J_{t}} \chi_{d}\right)-\frac{1}{\kappa} \int\left(r d \theta \hat{e}_{r}\right) \cdot\left(\vec{J}_{d} \chi_{1}\right)= \\
\frac{1}{\kappa} \int\left(r d \theta \hat{e}_{r}\right) \cdot\left(\vec{J}_{t} \chi_{d}\right)=-\frac{2}{\kappa} J_{t} d \int_{0}^{2 \pi} \cos \theta \sin (\theta-\phi) d \theta=-\frac{2 \pi}{\kappa}\left(\vec{J}_{t} \times \hat{e}_{z}\right) \cdot \vec{d} .
\end{aligned}
$$

The following term to solve is the first integral of the right side of the equation (26), which with the aid of the relation
(36) is written as follows

$$
\begin{aligned}
& \int d^{2} r P f_{0} f_{d} \\
& =\gamma_{2} \int_{0}^{\infty} \int_{0}^{2 \pi} d r d \theta\left\{v_{L} p_{1} \cos \left(\theta-\theta_{H}\right)+\right. \\
& \left.\quad v_{L} p_{2} \sin \left(\theta-\theta_{H}\right)-\varepsilon|\vec{\nabla} T| r \cos \theta\right\} f_{0} f_{d} .
\end{aligned}
$$


Developing the product $f_{0} f_{d}$ and using the expression for $\vec{d}$, given in equation (30), we have

$$
\begin{aligned}
& \gamma_{2} \int d^{2} r P f_{0} f_{d} \\
= & -\frac{\gamma_{2} \pi}{2}\left(\vec{v}_{L} \times \hat{e}_{z}\right) \cdot \vec{d} \int_{0}^{\infty}\left(f_{0}^{2}\right)^{\prime} r p_{1} d r-\frac{\gamma_{2} \pi}{2} \vec{v}_{L} \cdot \vec{d} \int_{0}^{\infty}\left(f_{0}^{2}\right)^{\prime} r p_{2} d r \\
& \quad-\pi \gamma_{2} \varepsilon \vec{\nabla} T \cdot \vec{d} \int_{0}^{\infty}\left(f_{0}^{2}\right)^{\prime} r^{2} d r .
\end{aligned}
$$

Solving the second integral in the right side of the equation (26), one must account the relations (12) and (15), obtaining

$$
\begin{aligned}
& \gamma_{1} \int d^{2} r f_{v} f_{d}=\gamma_{1} \int d^{2} r\left(\vec{v}_{L} \cdot \vec{\nabla} f_{0}\right)\left(\vec{d} \cdot \vec{\nabla} f_{0}\right) \\
&=-\pi \vec{v}_{L} \cdot \vec{d} \gamma_{1} \int_{0}^{\infty}\left(f_{0}^{\prime}\right)^{2} r d r
\end{aligned}
$$

where we have introduced the projections for $\vec{v}_{L}$ and $\vec{d}$ given by the equations (29) and (30) respectively. Now to calculate the third term of the equation (26), we expand $\rho_{n}(\vec{r})$ in a Taylor's series around the vortex centre

$$
\rho_{n}(\vec{r})=\rho_{n}(0)+\vec{r} \cdot \vec{\nabla} \rho_{n}(0)+\frac{(\vec{r} \cdot \vec{\nabla})^{2}}{2} \rho_{n}(0)+\ldots
$$

Accounting that, the position vector in cylindrical coordinates has the form $\vec{r}=r\left[\sin \left(\theta-\theta_{H}\right) \hat{e}_{r}+\cos \left(\theta-\theta_{H}\right) \hat{e}_{\theta}\right]$ and taking the scalar product, the function $\rho_{n}(\vec{r})$ can be written in the following way

$$
\begin{aligned}
\rho_{n}(\vec{r})=\rho_{n}(0)+ & r \sin \left(\theta-\theta_{H}\right) \frac{\partial}{\partial r} \rho_{n}(0) \\
& +\cos \left(\theta-\theta_{H}\right) \frac{\partial}{\partial \theta} \rho_{n}(0)+ \\
& \frac{r \frac{\partial}{\partial r} \rho_{n}(0)}{2}-\frac{r \sin \left(\theta-\theta_{H}\right) \cos \left(\theta-\theta_{H}\right)}{2} .
\end{aligned}
$$

Taking the gradient of the function (42) in the limit $r \rightarrow 0$, we obtain:

$$
\vec{\nabla} \rho_{n}(\vec{r})=\frac{\partial \rho_{n}(0)}{\partial r}\left(\hat{e}_{r}+\hat{e}_{\theta}\right)
$$

where we have considered that $\rho_{n}(\vec{r})$ also grows in the $-\hat{e}_{r}$ direction. Replacing the equation (43) in the solvability condition, the third term of this expression acquires the form:

$\frac{1}{\kappa} \int d^{2} r \chi_{d} \vec{v}_{n} \cdot \vec{\nabla} \rho_{n}(\hat{r})=\frac{\pi}{2 \kappa}\left[\left(\vec{v}_{n} \times \hat{e}_{z}\right) \cdot \vec{d}\right] \rho_{n}(0)+\frac{\pi}{\kappa}\left[\vec{v}_{n} \cdot \vec{d}\right] \rho_{n}(0)$.

Equally, the term associated to $\vec{v}_{L}$ in the solvability condition is obtained as follows:

$$
\begin{aligned}
& \frac{1}{\kappa} \int d^{2} r \chi_{d} \vec{v}_{L} \cdot \vec{\nabla} \rho_{n}(\hat{r})=- \frac{d v_{n}}{2} \times \\
& {\left[\int_{0}^{2 \pi} \sin \left(\theta-\theta_{H}\right)\left[\sin \left(\theta-\theta_{H}\right)+\cos \left(\theta-\theta_{H}\right)\right] d \theta \int_{0}^{\infty} \frac{\partial}{\partial r} \rho_{n}(0) d r\right]=} \\
& \frac{\pi}{2 \kappa}\left[\vec{v}_{L} \times \hat{e}_{z} \cdot \vec{d}\right] \rho_{n}(0)+\frac{\pi}{\kappa}\left[\vec{v}_{L} \cdot \vec{d}\right] \rho_{n}(0) .
\end{aligned}
$$

Finally, the fourth integral in equation (26) is solved using the relations (37) and (29):

$$
\begin{aligned}
& \frac{1}{\kappa} \int d^{2} r \chi_{d} \vec{v}_{L} \cdot \vec{\nabla} \rho_{s}(\hat{r})= \\
&-\frac{d v_{L}}{\kappa} \int_{0}^{2 \pi} \sin (\theta-\phi) \sin \left(\theta-\theta_{H}\right) d \theta \int_{0}^{\infty} \frac{\partial \rho_{s}}{\partial r} d r \\
&=-\pi \rho_{s}\left[\left(\vec{v}_{L} \times \hat{e}_{z}\right) \cdot \vec{d}\right] .
\end{aligned}
$$

The equation of motion is obtained from the solvability condition when we replace the results obtained in equations (38), (40), (41), (44), (45) and (46). In a compact form the equation of motion can be written:

$$
\begin{aligned}
\rho_{s}\left[\left(\vec{v}_{s}-\vec{v}_{L}\right) \times \overrightarrow{\tilde{\kappa}}\right]=D\left(\vec{v}_{L}-\vec{v}_{n}\right) & +D^{\prime}\left[\hat{e}_{z} \times\left(\vec{v}_{L}-\vec{v}_{n}\right)\right]+d \vec{v}_{L} \\
& +d^{\prime}\left(\hat{e}_{z} \times \vec{v}_{L}\right)+S_{v} \vec{\nabla} T
\end{aligned}
$$

In the above equation, $\overrightarrow{\widetilde{\kappa}}$ is the quantum of circulation and we have taken account that $\vec{J}_{t}=\vec{J}_{s} / 2$ and $\vec{J}_{s}=\rho_{s} \vec{v}_{s}$. In addition we have defined the coefficients

$$
\begin{gathered}
D=-\tilde{\kappa} \rho_{n}, D^{\prime}=\tilde{\kappa} \rho_{n} \\
d=\kappa \tilde{\kappa} \gamma_{1} \int_{0}^{\infty}\left(f_{0}^{\prime}\right)^{2} r^{2} d r-\kappa \tilde{\kappa} \frac{\gamma_{2}}{2} \int_{0}^{\infty} p_{2}\left(f_{0}^{2}\right)^{\prime} r^{2} d r \\
d^{\prime}=\kappa \tilde{\kappa} \frac{\gamma_{2}}{2} \int_{0}^{\infty} p_{1}\left(f_{0}^{2}\right)^{\prime} r^{2} d r \\
S_{v}=2 \gamma_{2} \eta \tilde{\kappa} \kappa \int_{0}^{\infty}\left(f_{0}^{2}\right)^{\prime} r^{2} d r \\
\rho_{n}=\rho_{n}(0) .
\end{gathered}
$$

The equation (47) determines the vortex dynamics in type II superconductors in presence of a temperature gradient. Equations of this type have been reported in some works in a 
phenomenological way [11]. In the present development this equation is obtained in a natural way from the time dependent Ginzburg - Landau equation. The term of the left side of equation (47) represents the Magnus force, which is connected to the momentum transfer between the vortex and the the superfluid. The forces proportional to $D$ and $D^{\prime}$ are due to dispersion of quasi particles by the vortices, presenting a momentum transfer between the vortex moving with velocity $\vec{v}_{L}$ and the normal fluid moving with velocity $\vec{v}_{n}$. The transverse component of this interaction is the Iordanskii force. The forces proportional to $d$ and $d^{\prime}$, according to Kopnin and Kravtsov [15], are due to vortex relaxation processes associated to the vortex interaction impurities which are at rest in the lattice. The transverse component of this interaction is the Kopnin-Kravtsov force. The force proportional to $\vec{\nabla} T$ is connected to the vortex thermal flow with a transport entropy $S_{v}$. If a moving vortex transports entropy, it experiences a force in a temperature gradient. The excistence of this force is well known from experimental data $[12,13,14]$.

\section{CALCULATION OF THE COEFFICIENTS $d, d^{\prime}$ AND $S_{v}$}

In this section we will determine the explicit form for the coefficients, which are connected to relaxation processes in the vortex dispersion so as the entropy $S_{v}$ due to the thermal motion. In general, when the Ginzburg-Landau equation is solved, one take some conjectures about the profile of the order parameter inside the vortex. An approach of the variation of dimensionless order parameter, which we will use, was proposed by Brandt [16]. In the present analysis we will find solutions in the limit $r \rightarrow 0$ where the order parameter has the form

$$
\frac{f_{0}^{2}}{2} \simeq 1-e^{-\frac{r^{2}}{2 \xi_{0}^{2}}} \simeq \frac{r^{2}}{2 \xi_{0}^{2}}
$$

Where $\xi_{0}$ is the coherence length. Replacing the equation (53) in the equation (33), the following equation in cylindrical coordinates is obtained:

$$
\frac{\partial^{2} p_{1}}{\partial r^{2}}+\frac{1}{r} \frac{\partial p_{1}}{\partial r}-R r^{2} p_{1}=0
$$

where the parameter $R=\kappa^{2} \gamma_{1} \xi_{0}^{2} \sigma_{x x}^{(n)}$ was introduced. The differential equation (54) has a solution in power's series given by the expession $p_{1}(r)=\sum_{n=0}^{\infty} a_{n} r^{n+q}$, where $q=-1$. The asymptotic solution to the equation (54) must consider that:

$$
\begin{aligned}
P & =\Phi+\frac{\partial \chi}{\partial t}=\vec{v} \cdot \vec{\nabla} \chi \cos \left(\theta-\theta_{H}\right)=v \vec{\nabla} \chi \cos \left(\theta-\theta_{H}\right) \\
& \approx \frac{1}{r} v \cos \left(\theta-\theta_{H}\right)
\end{aligned}
$$

From the previous analysis $p_{1}(r) \approx 1 / r$ in the limit $r \rightarrow 0$. In the same way for the magnetic vector we have $Q_{0}(r) \approx$ $-\frac{1}{\kappa} \nabla \chi \approx-\frac{1}{k r}$. But on the other hand, $Q_{0}$ satisfies the relation $h_{0}=\vec{\nabla} \times \vec{Q}_{0}$, where $Q_{0}(r)=\frac{1}{2} h_{0}(0) r$ which determines the solution $Q_{0}(r)=-\frac{1}{k r}+\frac{1}{2} h_{0}(0) r$, where $h_{0}(0)$ is the magnetic field in the vortex center. In this sense, accounting the relation (55) in the limit $r \rightarrow 0$, then we have $a_{1}=0$ and therefore

$$
p_{1}(r) \approx \frac{1}{r}
$$

In order to determine $p_{2}(r)$ in the same way that for $p_{1}(r)$, we look to the solution of the equation (32), in power series and doing all the previous procedure one obtain

$$
p_{1}(r) \approx \frac{1}{r}-p_{2 c} r
$$

Now we are able to determine coefficients $d, d^{\prime}$ and $S_{v}$. Replacing the expressions (56) and (57) in the equations (49), (50) and (51) in the limit $r \rightarrow 0$ :

$$
\begin{gathered}
d=\kappa \tilde{\kappa} \gamma_{1} \int_{0}^{\infty}\left(f_{0}^{\prime}\right)^{2} r d r+\frac{\gamma_{2}}{2} \int_{0}^{\infty} p_{2}\left(f_{0}^{2}\right)^{\prime} r d r=\kappa\left(\gamma_{2}-\gamma_{1}\right) \rho_{s}, \\
d^{\prime}=-\kappa \tilde{\kappa} \frac{\gamma_{2}}{2} \int_{0}^{\infty} p_{1}\left(f_{0}^{2}\right)^{\prime} r d r=-\kappa \gamma_{2} \rho_{s}
\end{gathered}
$$

$$
S_{v}=2 \tilde{\kappa} \kappa \gamma_{2} \eta \int_{0}^{\infty}\left(f_{0}^{2}\right)^{\prime} r^{2} d r=\tilde{\kappa} \kappa \gamma_{2} \eta \sqrt{2 \pi} \xi_{o}
$$

From the above equations we observe that the parameter $d$, which is associated to the interaction with impurities, has a double nature, the first one is connected to the BardeenStephen mechanism of dissipation, whereas the second contribution is due to variations in the local charge density in the vortex interaction with impurities. The same effect occurs in the obtaining of the coefficient $d^{\prime}$. Equally it is important to notice that the transport entropy posses a direct relation to relaxation processes due to the condensation and Coopers pair breaking by different pairing potentials via vortex motion as it is shown in equation (60).

\section{SUMMARY}

In the present analysis, we have obtained a vortex dynamics equation in type-II superconductors. Following previous works by Dorsey [5], Gorkov and Kopnin [6] we developed a more general equation for the vortex motion in the frame of the time dependent Ginzburg-Landau Equation. After some assumptions, we derived a local solvability condition, which governs the vortex dynamics. In this sense, we calculated the explicit form for the force coefficients, which are the keys for the understanding of the balance equation due to vortex interactions with the environment. In this connection, we took into account the spatial distribution of the normal electrons in the vortex core. This fact allowed to obtain exact expressions for the coefficients $D$ and $D^{\prime}$, which characterize the interaction between vortices and normal electrons by their dispersion. On the other hand, the approximated expressions for the coefficients $d$ and $d^{\prime}$, which characterize the vortex interaction with impurities were obtained. The nature of these coefficients is connected to the energy dissipation due to local variations of the charge density in the vortex core by the electron pairing. The same source of dissipation is associated to the transport entropy in the temperature gradient. 


\section{Acknowledgements}

The Authors would like to thank the financial support granted by the Universidad del Atlántico.

[1] J.M. Harris, N.P. Ong and Y.F. Yan, Phys. Rev. Lett. 71, 1455 (1993).

[2] S.J.Hagen et al., Phys. Rev. B 47, 1064 (1993).

[3] J. Bardeen and M.J. Stephen, Phys. Rev. 140, A1197 (1965).

[4] P. Nizierese and W.F. Vinen, Philos. Mag. 14, 667 (1966).

[5] A.T. Dorsey, Phys. Rev. B 46, 8376 (1992).

[6] L.P. Gorkov and N.B. Kopnin, Sov. Phys. Usp. 18, 496 (1975).

[7] P. Ao and Thouless, Phys. Rev. Lett. 70, 2158 (1993)

[8] P. Ao and Q Niu, Phys. Rev. Lett. 76, 3758 (1996)

[9] G. Blatter, V.G. Geshkenbein and N.B. Kopnin, Phys. Rev B
59, $14663(1999)$

[10] N.B. Kopnin, Rep. Prog. Phys. 65, 1633 (2002)

[11] A. Freimuth and M. Zittartz, Phys. Rev. Lett. 84, 4978 (2000).

[12] R.P. Huebner, Supercond. Sci. Technol. 8, 189 (1995).

[13] T.T.M. Palstra et al., Phys. Rev Lett. 64, 3090 (1990).

[14] M. Zeh et al., Phys. Rev. Lett. 64, 3195 (1990).

[15] N.B. Kopnin and V.E. Kravtsov, JETF Lett. 23, 578 (1976).

[16] E.H. Brandt, J. Low Temp. Phys. 24, 709 (1977). 